to calculate the individual and joint effects of CCT and TD, and we calculated the interaction contrast (IC) to illustrate possible interaction between these effects.

Results The effect of CCT on uptake of PMTCT services was greater among women exhibiting high TD. The IC suggested a positive interactive effect between TD and the CCT intervention on uptake of PMTCT services (IC: 0.17; 95\% CI: $0.15,0.48)$. We observed no evidence of additive interaction between TD and the CCT intervention on retention or viral suppression.

Conclusion This CCT intervention may help mitigate the harmful effects of TD on uptake of PMTCT services, though this mechanism did not appear to play a role for retention or viral suppression. Alternative approaches could be developed to address the effects of TD on HIV-related outcomes.

Disclosure No significant relationships.

\section{P314 IDENTIFYING KEY STAKEHOLDERS AND THEIR ROLES IN THE INTEGRATION OF POCTS FOR STIS INTO CLINICAL SERVICES}

${ }^{1}$ Agata Pacho*, ${ }^{1}$ Emma Heming De-Allie, ${ }^{1}$ Martina Furegato, ${ }^{1} E m m a$ Harding-Esch, ${ }^{2} \mathrm{~S}$ Tariq Sadiq, 'Sebastian Fuller. 'St George's, University of London, Applied Diagnostic Research and Evaluation Unit, Institute for Infection and Immunity, London, UK; ${ }^{2}$ St George's University of London, Applied Diagnostic Research and Evaluation Unit (ADREU), Institute for Infection and Immunity, London, UK

10.1136/sextrans-2019-sti.426

Background Despite potential to positively impact patient management, sequelae, and patient perceptions of services, few point-of-care tests (POCTs) to diagnose sexually transmitted infections (STIs) have been implemented into sexual health services (SHSs). Qualitative, in-depth research can be used to identify who the decision-makers are for adopting and implementing STI-POCTs in UK SHSs, and better understand these decision-makers' roles in these processes.

Methods We conducted a secondary analysis of data collected in two studies (Precise and Facilitators to Adoption). Based on their self-identified role in the POCT adoption process, sexual healthcare professionals (HCPs) were invited to in-depth interviews and workshops where participant observation notes were taken. Using these data, we defined key stakeholders and, using a thematic approach in NVIVO 11, we explored the process of POCT integration into their services.

Results 8 SHS workshops took place in 2017 and 37 interviews in 11 UK SHS took place between 2015 and 2018. Participants included clinicians, nurses, clinic managers, laboratory staff and clinical commissioners. Lead clinicians and managers self-identified themselves as key stakeholders for the decision to purchase, while nurses self-identified themselves as change champions for POCT implementation following adoption. Although many participants considered senior clinical staff most likely to introduce and drive change, participants stressed the importance of engagement of all clinical staff, particularly when tackling resistance to change.

Conclusion Our data suggest that supportive interpersonal relationships, such as between clinical leads and commissioners when considering adoption, and between all levels of clinical staff during implementation, must be considered to ensure the successful integration of POCTs in SHSs.

Disclosure No significant relationships.

\section{P316 STRUCTURE-BASED DRUG DESIGN FOR NEISSERIA GONORRHOEAE, CHLAMYDIA TRACHOMATIS, AND MYCOPLASMA GENITALIUM}

${ }^{1}$ Kayleigh Barrett, ${ }^{1}$ Samantha Michaels, ${ }^{1}$ Edelmar Navaluna, 'Latha Siddaramaiah, ${ }^{2}$ Gwendolyn Wood, ${ }^{3}$ Isabelle Phan, ${ }^{4}$ Zhongsheng Zhang, ${ }^{3}$ Bart Staker, ${ }^{3}$ Sandhya Subramanian, ${ }^{5}$ Patricia Totten, ${ }^{6}$ Olusegun Soge, ${ }^{3}$ Peter Myler, ${ }^{1}$ Robert Suchland, ${ }^{1}$ Lynn Barrett, ${ }^{1}$ Wes Van Voorhis, ${ }^{4}$ Erkang Fan, ${ }^{1}$ Kayode Ojo*, ${ }^{1}$ Kevin Hybiske. ${ }^{1}$ University of Washington, Seattle, USA; ${ }^{2}$ Univ WA, Seattle, USA; ${ }^{3}$ Seattle Children's Research Institute, Seattle, USA; ${ }^{4}$ University of Washington, Biochemistry, Seattle, USA; ${ }^{5}$ University of Washington, Infectious Diseases, Seattle, USA; ${ }^{6}$ University of Washington, Global Health, Seattle, USA

\subsection{6/sextrans-2019-sti.427}

Background The UW-STI consortium seeks to develop novel antimicrobials for the treatment of syndromically similar infections caused by Neisseria gonorrhoeae (GC), Chlamydia trachomatis (CT), and Mycoplasma genitalium (MG).

Methods We utilize a structure-based validation pipeline embedded with a gated series of criteria for progressing druggable enzyme targets, and for identifying and advancing compounds active against these protein targets. The pipeline includes orthologous and essential enzyme target identification, structure determination, compound library screening, antimicrobial susceptibility testing, hit optimization, and chemicalgenetic target validation.

Results To date, we have identified over 80 enzyme candidates that are essential, single copy genes in both GC and MG; 7 GC structures, $1 \mathrm{CT}$ structure and $1 \mathrm{MG}$ structure have been solved by crystallography, and soluble expression has been achieved for 19 GC, 20 CT, and 3 MG recombinant enzymes. Several structures common to two bacteria have been solved including tryptophan-tRNA synthetase, lysyl-tRNA synthetase, and ribose-5-phosphate isomerase A/B. Phenylalanyl-tRNA synthetase (PheRS) is among our highest priority targets and is presented as a proof of concept for multi-organism drug development. PheRS is a validated drug target with divergence from its human counterpart, as modeled by the group. In lieu of crystal structures, the GC PheRS alpha and beta complex was modeled using the Rosetta software suite. Multiple cloning and expression strategies have been employed including surface mutations, solubility tags, engineered truncations, and co-expression of both subunits, in hopes of producing crystals. A PheRS001 inhibitor was synthesized from published literature and proved active against GC PheRS with an $\mathrm{IC}_{50}$ of 93 $\mathrm{nM}$, and in antimicrobial testing against all three bacteria: 120 $\mu \mathrm{g} / \mathrm{ml}$ (CT MIC) and $18 \mu \mathrm{g} / \mathrm{ml}$ (MG and GC MIC).

Conclusion PheRS is a promising example of our pipeline capabilities in our three-pronged approach to produce 5-10 therapeutic leads and aid in the global fight against antibiotic resistance in sexually-transmitted bacterial infections.

Disclosure No significant relationships. 